Mathie, Dennis

\title{
"Am schlimmsten erging es den Türken". Explorative Untersuchung des Wissens über die Türken und die Türkei zwischen 1919 und 1936 anhand von drei Schulbuchreihen
}

Lohmann, Ingrid [Hrsg.]; Böttcher, Julika [Hrsg.]: Türken- und Türkeibilder im 19. und 20. Jahrhundert. Pädagogik, Bildungspolitik, Kulturtransfer. Bad Heilbrunn : Verlag Julius Klinkhardt 2021, S. 119-142. - (Wie die Türken in unsere Köpfe kamen. Eine deutsche Bildungsgeschichte; 1)

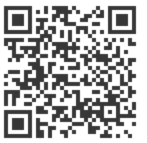

\section{Quellenangabe/ Reference:}

Mathie, Dennis: "Am schlimmsten erging es den Türken". Explorative Untersuchung des Wissens über die Türken und die Türkei zwischen 1919 und 1936 anhand von drei Schulbuchreihen - In: Lohmann, Ingrid [Hrsg.]; Böttcher, Julika [Hrsg.]: Türken- und Türkeibilder im 19. und 20.

Jahrhundert. Pädagogik, Bildungspolitik, Kulturtransfer. Bad Heilbrunn : Verlag Julius Klinkhardt 2021, S. 119-142 - URN: urn:nbn:de:0111-pedocs-219343 - DOI: 10.25656/01:21934

https://nbn-resolving.org/urn:nbn:de:0111-pedocs-219343

https://doi.org/10.25656/01:21934

in Kooperation mit / in cooperation with:

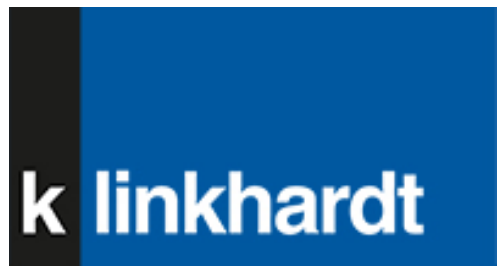

http://www.klinkhardt.de

\section{Nutzungsbedingungen}

Dieses Dokument steht unter folgender Creative Commons-Lizenz: http://creativecommons.org/licenses/by-nc-sa/4.0/deed.de - Sie dürfen das Werk bzw. den Inhalt unter folgenden Bedingungen vervielfältigen, verbreiten und öffentlich zugänglich machen sowie Abwandlungen und Bearbeitungen des Werkes bzw. Inhaltes anfertigen: Sie müssen den Namen des Autors/Rechteinhabers in der von ihm festgelegten Weise nennen. Dieses Werk bzw. der Inhalt darf nicht für kommerzielle Žwecke verwendet werden. Die neu entstandenen Werke bzw. Inhalte dürfen nur unter Verwendung von Lizenzbedingungen weitergegeben werden, die mit denen dieses Lizenzvertrages identisch oder vergleichbar sind.

Mit der Verwendung dieses Dokuments erkennen Sie die Nutzungsbedingungen an.

\section{Terms of use}

This document is published under following Creative Commons-License: http://creativecommons.org/licenses/by-nc-sa/4.0/deed.en - You may copy, distribute and transmit, adapt or exhibit the work in the public and alter, transform or change this work as long as you attribute the work in the manner specified by the author or licensor. You are not allowed to make commercial use of the work. If you alter, transform, or change this work in any way, you may distribute the resulting work only under this or a comparable license.

By using this particular document, you accept the above-stated conditions of use.

\section{Kontakt / Contact:}

\section{peDOCS}

DIPF | Leibniz-Institut für Bildungsforschung und Bildungsinformation

Informationszentrum (IZ) Bildung

E-Mail: pedocs@dipf.de

Internet: www.pedocs.de

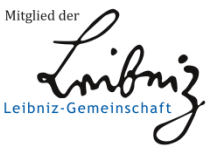




\title{
Wie die Türken in unsere Köpfe kamen. Eine deutsche Bildungsgeschichte
}

\author{
herausgegeben von \\ Ingrid Lohmann, Julika Böttcher, Christine Mayer \\ und Sylvia Kesper-Biermann
}

Band 1

Die Reihe versammelt interdisziplinäre Forschungsergebnisse und Quellentexte zu den deutsch-türkischen Bildungsbeziehungen im 19. und 20. Jahrhundert. Sie nimmt transnationale sowie postkoloniale Perspektiven ein und trägt so zum erweiterten Verständnis eines heute weitgehend unbekannten Kapitels der deutschen Bildungsgeschichte bei. Die Reihe zielt darauf ab, die Historiographie deutscher Pädagogik zu erweitern und das Selbstverständnis der im Bildungswesen Tätigen zu hinterfragen. 
Ingrid Lohmann Julika Böttcher

(Hrsg.)

\section{Türken- und Türkeibilder im 19. und 20. Jahrhundert}

Pädagogik, Bildungspolitik, Kulturtransfer 
Dieser Titel wurde in das Programm des Verlages mittels eines Peer-Review-Verfahrens aufgenommen. Für weitere Informationen siehe www.klinkhardt.de.

Bibliografische Information der Deutschen Nationalbibliothek Die Deutsche Nationalbibliothek verzeichnet diese Publikation in der Deutschen Nationalbibliografie; detaillierte bibliografische Daten sind im Internet abrufbar über http://dnb.d-nb.de.

2021.lg. (C) by Julius Klinkhardt.

Abbildung Umschlagseite 1: Der Schachtürke, nach dem Kupferstich in Joseph Friedrich zu Racknitz: Ueber den Schachspieler des Herrn von Kempelen und dessen Nachbildung. Leipzig: Breitkopf 1789, Tafel III.

Digitale Sammlungen der Humboldt-Universität zu Berlin, urn:nbn:de:kobv:11-d-4709479.

Druck und Bindung: AZ Druck und Datentechnik, Kempten.

Printed in Germany 2021.

Gedruckt auf chlorfrei gebleichtem alterungsbeständigem Papier.

Die Publikation (mit Ausnahme aller Fotos, Grafiken und Abbildungen) ist veröffent-

licht unter der Creative Commons-Lizenz: CC BY-NC-SA 4.0 International

https://creativecommons.org/licenses/by-nc-sa/4.0/

ISBN 978-3-7815-5874-8 digital doi.org/10.35468/5874

ISBN 978-3-7815-2436-1 


\section{Inhaltsverzeichnis}

Ingrid Lohmann, Julika Böttcher

Einleitung und Dank

\section{Diskurse und Wandlungsprozesse}

Fikret Adanır

Wandlungen des deutschen Türkeibildes in der ersten Hälfte

des 20. Jahrhunderts

Ingrid Lohmann

Lieber Türken als Levantiner -

Deutsche Kolonialinteressen an der Türkei und die Stimme

der Pädagogik im langen 19. Jahrhundert

\section{Julika Böttcher}

Die Türkei als Argument in der pädagogischen Presse des

Wilhelminischen Kaiserreichs

\section{Schulbuchanalysen}

Timm Gerd Hellmanzik

„Als Brüder sind die Türken niemals betrachtet, auch der europäischen

Geistesbildung immer fremd geblieben“ - Das Türkenbild in Joseph Annegarns

Weltgeschichte für die katholische Jugend (1840)

Dennis Mathie

„Am schlimmsten erging es den Türken“-

Explorative Untersuchung des Wissens über die Türken und die Türkei

zwischen 1919 und 1936 anhand von drei Schulbuchreihen. 
Andreas Hoffmann-Ocon, Norbert Grube

„Wo die Parteien sich mit Staatsstreichen, Militärrevolten, Hinrichtungen und Morden bekämpfen " - Türkeibilder in schweizerischen

Geschichtslehrmitteln in der ersten Hälfte des 20. Jahrhunderts

\section{Experten und Kulturtransfer}

Filiz Meşeci Giorgetti

Reformpädagogik in türkischen Elementarschulen. Eine Untersuchung zum türkisch-deutschen Austausch über Erziehung und Unterricht in der ersten Hälfte des 20. Jahrhunderts

Christine Mayer

Zur Formierung einer transnationalen Sphäre durch Bildungsexperten John Deweys Reise in die Türkei 1924

\section{Christian Roith}

Pädagogische Modernisierungsstrategien in Spanien

zu Beginn des 20. Jahrhunderts

Sebastian Willert

„Hamdi hat hier gewütet \& da gewühlt“ -

Perspektiven deutscher Forschungsreisender auf die Anfänge der osmanischen Archäologie, 1881-1892

Über die Autorinnen und Autoren .275 


\section{„Am schlimmsten erging es den Türken“ - Explorative Untersuchung des Wissens über die Türken und die Türkei zwischen 1919 und 1936 anhand von drei Schulbuchreihen}

Zusammenfassung: Der vorliegende Beitrag untersucht die Konstruktion des Wissens über die Türken und die Türkei für das Unterrichtsfach Geschichte. Die ausgewählten Werke erschienen zwischen 1923 und 1936. In diesem Zeitraum waren die deutschtürkischen Beziehungen von einem wechselvollen Prozess der Abgrenzung und der Annäherung gekennzeichnet. Gleichzeitig wurde in beiden Staaten eine neue, als revolutionär empfundene Regierungsform etabliert. Auch das Wissen über den Anderen unterlag einem Neu- und Restrukturierungsprozess. Ein maßgeblicher Teil dieses Prozesses sind Diskurse im Sinne Michel Foucaults. Sie werden im Folgenden exemplarisch anhand von drei mehrfach aufgelegten und weit verbreiteten Schulbuchreihen analysiert, und zwar im Hinblick auf narrative Strukturen, die für die Produktion des themenspezifischen Wissens bedeutsam waren: erstens der Topos der "grausamen türkischen Herrschaft", zweitens das Narrativ des Niedergangs, mit welchem die im 19. Jahrhundert erlittenen territorialen Verluste des Osmanischen Reiches dargestellt wurden, drittens dessen fragliche Zugehörigkeit zu Europa, viertens das Narrativ der zivilisatorischen Überlegenheit des Deutschen Reiches. Wie sich zeigt, bleiben traditionelle Zuschreibungen und überkommene historische Darstellungen zunächst unverändert. Erst in der Spätphase der Weimarer Republik und zu Beginn des Nationalsozialismus zeigt sich ein tendenzieller Wandel der Narrative.

\section{Einleitung}

Der Trauerzug für den ehemaligen osmanischen Großwesir Mehmed Talat Paşa (1874-1921) in Berlin im März 1921 symbolisierte das Ende der Verbindung zwischen dem Deutschen und dem Osmanischen Reich nach dem verlorenen Ersten Weltkrieg. Die Kriegsniederlage war, wie Mangold-Will und Guillemarre-Acet herausgearbeitet haben, auch der Ausgangspunkt einer neuen deutsch-türkischen Zusammenarbeit, die nicht mehr als Fortsetzungsgeschichte der bisherigen Freundschaft zu bewerten sei, sondern differenziert betrachtet werden 
müsse. ${ }^{1}$ Obwohl die Siegermächte die Beziehungen der beiden Reiche unterbunden hatten, gab es weiterhin Befürworter einer besonderen Verbindung zwischen ihnen, vor allem in national-konservativen und völkischen Gruppierungen. ${ }^{2} \mathrm{Zu}$ diesen gehörte der Bund der Asienkämpfer, die wichtigste Vereinigung der ehemals im osmanischen Heer aktiven deutschen Soldaten und Offiziere des Ersten Weltkriegs. ${ }^{3}$ Die offizielle außenpolitische Diktion der Weimarer Republik hingegen prägten „Formulierungen von der traditionellen deutsch-türkischen Freundschaft und dem politischen Desinteresse “4 Zudem habe der politische Republikanismus, so Mangold-Will, generell nur geringes Interesse an der entstehenden Türkischen Republik und der kemalistischen Gesellschaft entwickelt, und Ereignisse in der Türkei seien als wenig bedeutsam für die deutsche Öffentlichkeit, sondern als innertürkische Angelegenheit angesehen worden. ${ }^{5}$ Das zunächst verbindende Element der gemeinsamen Kriegsniederlage und der als ungerechtfertigt betrachteten Friedensverträge von Versailles (1919), Sèvres (1920) und Lausanne (1923) war überdies aus türkischer Sicht aufgrund des positiven Ausgangs des Türkischen Unabhängigkeitskrieges nicht mehr relevant. ${ }^{6}$ Auch die Imagination eines deutsch-türkischen Wirtschafts- und Kulturraums ${ }^{7}$ verlor in der Weimarer Zeit erheblich an Bedeutung. Aus dem pädagogischen Diskurssegment verschwand die Türkei trotzdem nicht. Mit dem Machtantritt der Nationalsozialisten 1933 gewann eine neue Deutung der neuen deutschen und türkischen Staatsformen Relevanz: Behauptet wurde eine wechselseitige Verbundenheit aufgrund einer Gleichförmigkeit der Entwicklung des Kemalismus und des nationalsozialistischen Führerstaats. ${ }^{8}$

Die Spezifik des Medientyps Schulbuch sowie des in Schulbüchern repräsentierten Wissens ermöglicht eine diskursanalytische Betrachtung. Diese richtet sich mit Foucault darauf, was in einem spezifischen historischen Zeitraum sagbar

1 Vgl. Guillemarre-Acet, Dorothée: Impérialisme et nationalisme. L’Allemagne, l'Empire ottoman et la Turquie (1908-1933). Würzburg: Ergon 2009; Mangold-Will, Sabine: Begrenzte Freundschaft: Deutschland und die Türkei 1918-1933. Göttingen: Wallstein 2013.

2 Vgl. ebd. S. 499ff; Ihrig, Stefan: Atatürk in the Nazi Imagination. Cambridge: The Belknap Press of Harvard University Press 2014.

3 Vgl. Riedler, Florian: Nationalismus und internationale Sensibilität. Transnationale Akteure und die deutsch-türkischen Beziehungen in der Zwischenkriegszeit. In: Aufbruch ins postkoloniale Zeitalter. Hrsg. von Sönke Kunkel und Christoph Meyer. Frankfurt am Main: Campus 2012, S. 251-274.

4 Mangold-Will: Begrenzte Freundschaft, 2013, S. 500.

5 Vgl. ebd. S. 501.

6 Vgl. Leonhard, Jörn: Der überforderte Frieden: Versailles und die Welt 1918-1923. München: Beck 2018.

7 Vgl. Böttcher, Julika; Kesper-Biermann, Sylvia; Lohmann, Ingrid; Mayer, Christine: Deutsch-türkischer Bildungsraum um 1918 - Akteure, Visionen und Transformationen. In: Jahrbuch für Historische Bildungsforschung 25.1 (2019), S. 114-159.

8 Vgl. Ihrig: Atatürk in the Nazi Imagination, 2014, S. 202. 
und wissbar ist, ${ }^{9}$ wobei im vorliegenden Fall selbst die Benennungen „Türkei“ und „Türken“ Teil einer diskursiven Konstruktion waren. ${ }^{10}$ In den Quellen sind „Türkei“ und „Osmanisches Reich“ synonym. So wird in einem Abschnitt mit dem Titel „Die Angriffe der mohammedanischen Staaten“ festgehalten: „Das Osmanenreich war um die Mitte des 16. Jahrhunderts auf der Höhe seiner Macht. [...] Die französischen Könige hielten an ihrer Bündnispolitik mit der Türkei gegen die Habsburger fest. [...] Im Mittelmeere bedrohten die Türken die venetianischen Besitzungen im Ägäischen Meere "11. Auch die Benennungen „Osmanen“ und „Türken“ wurden unabhängig vom dargestellten Kontext durchweg synonym verwendet. Allerdings lässt sich eine klare Präferenz für die Verwendung des Wortfeldes „Türkei“ erkennen:

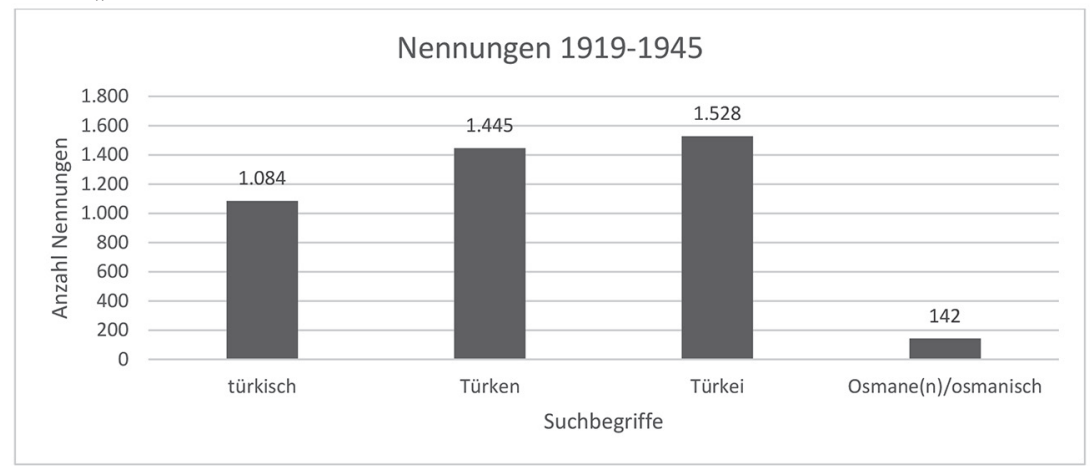

Abb.: Quantitative Untersuchung des Gesamtkorpus der Lehrbücher auf Basis der Suchworte türk*, osman*

Der vorliegende Beitrag untersucht anhand von drei Schulbuchreihen, welches Wissen über Türken und die Türkei sie enthalten - und in welchen narrativen Strukturen -, auch im Hinblick darauf, ob und gegebenenfalls welche Änderungen im Nachklang an den Wechsel der Regierungsformen darin einflossen. Die ausgewählten Werke eignen sich für die Analyse in besonderer Weise, da sie in den ersten Auflagen vor der großen Lehrplanrevision der Weimarer Republik erschienen, sodann die aktuellen historischen Veränderungen aufnahmen und

9 Vgl. Link, Jürgen: Diskurs, Interdiskurs, Kollektivsymbolik. Am Beispiel der aktuellen Krise der Normalität. In: Zeitschrift für Diskursforschung 1.1 (2013), S. 7-23, hier 9.

10 „Die Gleichsetzung von Türken mit Osmanen oder der Türkei mit dem Osmanischen Staat bzw. dem Osmanischen Reich ist zwar nicht völlig angemessen, aber durch jahrhundertelangen westlichen und östlichen Gebrauch sanktioniert." Kreiser, Klaus: Der osmanische Staat 1300-1922. München: Oldenbourg 2008, S. 3. Im vorliegenden Artikel wird die jeweilige zeitgenössische Bezeichnung gewählt.

11 Schiefer, Wilhelm; Hohmann, Walter; Maier, Albert; Schirmeyer, Ludwig: Deutsche Geschichte vom Spätmittelalter (1250) bis zum Wiener Kongreß (1815). Frankfurt am Main: Diesterweg 1935, S. 52. 
schließlich 1935, zwei Jahre nach dem Machtantritt der Nationalsozialisten, letzte Auflagen erfuhren. Die Untersuchung konzentriert sich auf jene Bände der Reihe, die den Zeitraum zwischen dem frühen 19. Jahrhundert und ihrer eigenen Gegenwart behandeln. Damit lässt sich ein ausreichend langer Zeitraum, in dem gesellschaftliche Veränderungen in das ,träge Wissen “ der Schulbücher einwirkten, in den Blick nehmen. ${ }^{12}$

\section{Schulbücher, Wissen und narrative Strukturen}

Geschichtsschulbücher des Untersuchungszeitraums benennen, unabhängig davon, ob sie als Tabelle, Quellenbuch oder erzählende Darstellung konzipiert sind, eine Abfolge von Ereignissen. Entsprechend ist Wissen, ebenso wie Nicht-Wissen, in besonderem Maße an die dargestellten Ereignisse gekoppelt. Auch bei der Schilderung bestimmter Perioden, etwa von Regierungsphasen einzelner Herrscher, können in den Schulbüchern unterschiedliche bedeutungstragende Elemente herangezogen werden. Daher muss im Sinne Foucaults zunächst positivistisch das in ihnen (re-)präsentierte Wissen erfasst werden, erst anschließend kann dargelegt werden, in welche narrativen Strukturen es eingebunden ist. Hierunter versteht Keller etwa die „story lines, roten Fäden, plots oder narrative Muster, durch welche die einzelnen Bestandteile einer Aussage zu einer kleineren oder größeren Erzählung bzw. Geschichte verbunden, also über die willkürliche Reihung sprachlicher Äußerungen hinaus spezifisch konfiguriert werden “13. Viehöver ergänzt, dass narrativen Strukturen nicht nur eine Funktion als Wissenstransporteuren zukommt, sondern dass sie in Anlehnung an Hayden White als ein „impulse to moralize reality“ ${ }^{14}$ zu verstehen sind. Dabei ist das in Schulbüchern rekonstruierte Wissen bereits eine spezifische Form, Wissen anzusprechen. Höhne zufolge kann es durch die Metaphern der jeweiligen Diskursarena und die netzförmige Anbindung an andere Wissens- und Diskursbereiche erfasst werden, und es tritt selbst diskursförmig auf. ${ }^{15}$

In den Blick genommen werden Teilbände von drei der renommiertesten Lehrbuchreihen der Weimarer Republik. Der Teubner-Verlag gab ab 1923 die Reihen Lehrbuch der Geschichte für die Mittelstufe sowie Grundriß der Geschichte für die

12 Vgl. Höhne, Thomas: Schulbuchwissen. Frankfurt am Main: Johann Wolfgang Goethe-Universität 2003, S. $102 \mathrm{f}$.

13 Keller, Reiner: Diskursforschung. Eine Einführung für SozialwissenschaftlerInnen. Wiesbaden: VS Verlag für Sozialforschungen 2011, S. 110.

14 White, Hayden: The Value of Narrativity in the Representation of Reality. In: Critical Inquiry 7.1 (1980), S. 5-27, hier 18; Viehöver, Willy: Diskurse als Narrationen. In: Handbuch sozialwissenschaftliche Diskursanalyse: Bd. 1 Theorien und Methoden. Hrsg. von Reiner Keller. Wiesbaden: VS Verlag für Sozialwissenschaften 2006, S. 179-208, hier 184.

15 Vgl. dazu grundsätzlich Höhne: Schulbuchwissen, 2003. 
Oberstufe höherer Lehranstalten heraus. Ab dem gleichen Zeitraum erschienen in der Halle'schen Buchhandlung des Waisenhauses die Grundzüge der Geschichte für höhere Lehranstalten für die Unter-, die Mittel- und die Oberstufe. Die drei, vier und acht Bände umfassenden Reihen boten eine Grundlage für den Geschichtsunterricht für alle höheren Schulformen und erlebten bis 1936 wiederholt Neuauflagen.

Die Schulbücher wurden zunächst für Preußen konzipiert und zugelassen, dann aber auch in weiteren Gebieten der Weimarer Republik verwendet. ${ }^{16}$ Konzeptionell unterscheiden sich die Publikationen schon insofern, als für den Teubner-Verlag unterschiedliche Autoren tätig waren, während Friedrich Neubauer alle Einzelbände der Grundzüge besorgte. ${ }^{17}$ Neubauer (1861-1953), promovierter Lehrer und langjähriger Direktor des Lessing-Gymnasiums in Frankfurt am Main, zählte zu den bekanntesten und auflagenstärksten Autoren der Kaiserzeit sowie der frühen Weimarer Republik. ${ }^{18}$ Als Verfasser des Mittelstufenbandes im Teubner-Verlag trat Hermann Pinnow (1881-1973) hervor, der ebenfalls in Geschichte promoviert worden und langjährig im höheren Schuldienst tätig war; die Veröffentlichung von 1923 war sein erstes Schulbuch. ${ }^{19}$ Franz Schnabel (1887-1966), der Autor des Oberstufenbandes, lehrte von 1922 bis 1936, dem Jahr, in dem er aus politischen Gründen aus dem Universitätsdienst entlassen wurde, an der Technischen Hochschule Karlsruhe; nach 1945 war er Lehrstuhlinhaber für Mittlere und Neuere Geschichte in München. ${ }^{20}$

16 Vgl. Blänsdorf, Agnes: Lehrwerke für Geschichtsunterricht an Höheren Schulen 1933-1945: Autoren und Verlage unter den Bedingungen des Nationalsozialismus. In: Nationalsozialismus in den Kulturwissenschaften. Band 1: Fächer - Milieus - Karrieren. Hrsg. von Hertmut Lehmann, Otto Gerhard Oexle. Göttingen: Vandenhoeck \& Ruprecht 2004, S. 273-370, hier 317f. Die Bezeichnung „Weimarer Republik” verbreitete sich zunächst als Negativfolie in republikfeindlichen Gruppierungen, setzte sich aber nach dem Zweiten Weltkrieg als namentliche Unterscheidung durch. Vgl. Ullrich, Sebastian: Mehr Schall als Rauch. Der Streit um den Namen der ersten deutschen Demokratie 1918-1949. In: Die „Krise“ der Weimarer Republik: zur Kritik eines Deutungsmusters. Hrsg. von Moritz Föllmer. Frankfurt am Main: Campus 2005, S. 187-208.

17 Zur Provenienz der Autoren von Geschichtsschulbüchern vgl. Jacobmeyer, Wolfgang: Das deutsche Schulgeschichtsbuch 1700-1945. Die erste Epoche seiner Gattungsgeschichte im Spiegel der Vorworte. Berlin: LIT 2011.

18 Vgl. Kreusch, Julia: Der Verlag der Buchhandlung des Waisenhauses als Schulbuchverlag zwischen 1830 und 1918. Die erfolgreichen Geografie- und Geschichtslehrbücher und ihre Autoren. Halle: Verlag der Franckeschen Stiftungen Halle im Max Niemeyer Verlag 2008, S. $211 \mathrm{ff}$.

19 Vgl. Jacobmeyer: Das deutsche Schulgeschichtsbuch 1700-1945, 2011, S. 1402, Anm. 18.

20 Vgl. Hertfelder, Thomas: Franz Schnabel und die deutsche Geschichtswissenschaft. Geschichtsschreibung zwischen Historismus und Kulturkritik (1910-1945). Göttingen: Vandenhoeck \& Ruprecht 1998, S. 614-689. 


\section{Dennis Mathie}

\begin{tabular}{|c|c|c|c|}
\hline Reihentitel & Schulbuchtitel & Autor(en) & Erstausgabe \\
\hline \multirow{3}{*}{$\begin{array}{l}\text { Lehrbuch der } \\
\text { Geschichte } \\
\text { für die } \\
\text { Mittelstufe }\end{array}$} & $\begin{array}{l}\text { Geschichte des } \\
\text { Altertums }\end{array}$ & Pinnow, Hermann & 1923 \\
\hline & $\begin{array}{l}\text { Geschichte des } \\
\text { deutschen Volkes von } \\
\text { den Anfängen bis } 1648\end{array}$ & Pinnow, Hermann & 1923 \\
\hline & $\begin{array}{l}\text { Geschichte des } \\
\text { deutschen Volkes von } \\
1648 \text { bis zur Gegenwart }\end{array}$ & Pinnow, Hermann & 1923 \\
\hline \multirow{4}{*}{$\begin{array}{l}\text { Grundriß der } \\
\text { Geschichte } \\
\text { für die } \\
\text { Oberstufe } \\
\text { höherer } \\
\text { Lehranstalten }\end{array}$} & Altertum & Neustadt, Ernst; Röhm, Georg & 1924 \\
\hline & Mittelalter & Bonwetsch, Gerhard & 1924 \\
\hline & Neuzeit & Bonwetsch, Gerhard; Kania, Hans & 1924 \\
\hline & Neueste Zeit & Schnabel, Franz & 1924 \\
\hline \multirow{8}{*}{$\begin{array}{l}\text { Grundzüge } \\
\text { der } \\
\text { Geschichte } \\
\text { für höhere } \\
\text { Lehranstalten }\end{array}$} & $\begin{array}{l}\text { Deutsche Geschichte } \\
\text { seit Anfang des } 18 . \\
\text { Jahrhunderts für die } \\
\text { Unterstufe }\end{array}$ & Neubauer, Friedrich & 1923 \\
\hline & $\begin{array}{l}\text { Geschichte des } \\
\text { Altertums und des } \\
\text { frühen Mittelalters für } \\
\text { die Mittelstufe }\end{array}$ & Neubauer, Friedrich & 1923 \\
\hline & $\begin{array}{l}\text { Deutsche Geschichte } \\
\text { von der Gründung } \\
\text { des nationalen Staats } \\
\text { bis zum Westfälischen } \\
\text { Frieden für die } \\
\text { Mittelstufe }\end{array}$ & Neubauer, Friedrich & 1923 \\
\hline & $\begin{array}{l}\text { Vom Westfälischen } \\
\text { Frieden bis zum } \\
\text { Wiener Kongreß für die } \\
\text { Mittelstufe }\end{array}$ & Neubauer, Friedrich & 1923 \\
\hline & $\begin{array}{l}\text { Vom Wiener Kongreß } \\
\text { bis zur Gegenwart für } \\
\text { die Mittelstufe }\end{array}$ & Neubauer, Friedrich & 1923 \\
\hline & $\begin{array}{l}\text { Geschichte des } \\
\text { Altertums und des } \\
\text { frühen Mittelalters für } \\
\text { die Oberstufe }\end{array}$ & Neubauer, Friedrich & 1923 \\
\hline & $\begin{array}{l}\text { Von der Gründung des } \\
\text { deutschen nationalen } \\
\text { Staates bis zum Ende des } \\
\text { 18. Jahrhunderts für die } \\
\text { Oberstufe }\end{array}$ & Neubauer, Friedrich & 1924 \\
\hline & $\begin{array}{l}\text { Vom Beginn des } 19 . \\
\text { Jahrhunderts bis zur } \\
\text { Gegenwart für die } \\
\text { Oberstufe }\end{array}$ & Neubauer, Friedrich & 1924 \\
\hline
\end{tabular}


Geschichtsschulbücher stehen und entstehen in einem vielschichtigen diskursiven Netz. Die Kontextualisierung der einzelnen Werke sowie übergreifende Elemente sind Bestandteile historischer und wissenssoziologischer Diskursanalyse. ${ }^{21}$ Eines dieser Elemente ist die Lehrplananbindung und Zulassungspraxis. So wurde beispielsweise in Preußen 1913 verordnet, dass sich neu eingeführte Lehrbücher an den amtlichen Lehrplänen und Lehraufgaben zu orientieren hätten. ${ }^{22} \mathrm{Nach}$ dem Ende des Ersten Weltkrieges erfolgte am 6. Dezember 1919 zunächst das Verbot aller bis dahin zugelassenen Geschichtsschulbücher, das aber nach umfassenden Protesten von Lehrkräften und Schulbuchproduzenten bereits am 8. April 1920 teilweise zurückgenommen wurde. ${ }^{23}$ Drei Jahre später, im September 1923, kam es schließlich zu einer Neuregelung der Zulassungspraxis. Die Verlage konnten nun erstmals bei den Provinzialschulkollegien die Zulassung beantragen. ${ }^{24}$ Die Erstauflagen der hier untersuchten Schulbuchreihen waren einige der ersten Werke, die im Rahmen des neuen Zulassungsverfahrens, aber noch vor der Einführung neuer Lehrpläne erschienen. Deren endgültige Fassung wurde 1925 in der Form von Richtlinien veröffentlicht. Sämtliche hier untersuchten Schulbücher wurden daraufhin umgehend überarbeitet. So heißt es noch in der siebten Auflage, 1930, von Schnabels Beitrag zum Grundriß: „[Daß] die Forderungen der preußischen Richtlinien für die Lehrpläne von 1925 für den weiteren Ausbau des Buches grundlegend waren, daß aber auch die Lehrpläne der anderen deutschen Länder und die zahlreichen wertvollen theoretischen Erörterungen der Fachkreise sorgfältig beachtet wurden, bedarf wohl kaum eines besonderen Hinweises“25. Die enge Verzahnung von Lehrplan und Lehrbuchinhalten entspricht der Tendenz zur Vereinheitlichung des Lehrbuchsektors, die seit dem späten 19. Jahrhundert beobachtet werden kann. In den Lehrplänen wird die Behandlung der deutschen Geschichte in den Vordergrund gerückt, jedoch soll besonders in der

21 Vgl. Landwehr, Achim: Historische Diskursanalyse. Frankfurt am Main: Campus 2009, sowie Keller, Reiner: Wissenssoziologische Diskursanalyse. In: Handbuch sozialwissenschaftliche Diskursanalyse. Hrsg. von Reiner Keller, Andreas Hirseland, Werner Schneider et al. Wiesbaden: VS Verlag 2011, S. 125-158.

22 Vgl. Ordnung für die Einführung von Lehrbüchern an den höheren Lehranstalten für die männliche und für die weibliche Jugend. Berlin: Zentralblatt für die gesamte Unterrichtsverwaltung 1913. Vgl. Kreusch, Julia: Der Schulbuchverlag. In: Geschichte des deutschen Buchhandels im 19. und 20. Jahrhundert. Die Weimarer Republik 1918-1933. Hrsg. von der Historischer Kommission des Börsenvereins des Deutschen Buchhandels. Berlin: de Gruyter 2012, S. 219-240, hier 219. - Für die Umsetzung der Verordnung in einzelnen Verlagen vgl. Kreusch: Der Verlag der Buchhandlung des Waisenhauses, 2008, S. 37ff. Ähnliche Erlasse lassen sich für die anderen Länder des Deutschen Reiches finden.

23 Vgl. Schellberg, Wilhelm; Hylla, Erich: Die Bestimmungen über Einführung von Lehrbüchern. Berlin: Weidmannsche Buchhandlung 1926, S. 53 f.

24 Vgl. Kreusch: Weimarer Republik, 2012, S. 224.

25 Schnabel, Franz: Neueste Zeit. Leipzig: Teubner 1930, S. V. 
Oberstufe auch die „Geschichte anderer Nationen behandelt werden“, mit dem Ziel, „die großen Leistungen auch der anderen Völker erkennen und würdigen zu lernen“26. Die Türken werden nur einmal explizit genannt, nämlich wenn „das Aufkommen Österreichs als Großmacht; seine weltgeschichtliche Bedeutung als Bollwerk gegen die Türken “27 behandelt wird. Das Gebiet, das nach 1871 das Deutsche Reich bildete, sollte „besondere Berücksichtigung “" ${ }^{28}$ finden. Auch nach dem Machtantritt der Nationalsozialisten wurden zunächst weder die Lehrpläne noch die Zulassungspraxis verändert. Die Lehrbuchproduzenten agierten in den ersten Jahren der nationalsozialistischen Herrschaft in einer unklaren Situation, in der sowohl eine Anpassung an die neuen Machtverhältnisse als auch ein unverändertes Beibehalten der bestehenden Schulbuchreihen versucht wurde. So heißt es in einem Vorwort von 1935:

„Solange jedoch keine bindenden Vorschriften für die Neuordnung der höheren Schule vorliegen, dürfte es nicht zweckmäßig sein, die Schulbücher grundlegend zu ändern. Auf Anordnung und mit Genehmigung des Reichsministeriums für Erziehung und Unterricht wurde daher eine Zwischenlösung gesucht. Ergänzungsbogen [...] sollen es dem Unterricht ermöglichen auf der Grundlage des vorliegenden Buchtextes den Gegenwartsaufgaben unseres Volkes und Staates zu entsprechen." ${ }^{\text {"29 }}$

Die insoweit skizzierte Praxis der Schulbuchproduktion zwischen Lehrplananbindung und Zulassungskriterien macht zwar die Entstehung der einzelnen Werke nicht kleinschrittig nachvollziehbar, doch wird in den Vorworten wiederholt betont, dass eine enge Anbindung an das bestehende Regelwerk erfolgt sei. Von daher lässt sich das in ihnen enthaltene Wissen als eines verstehen, das zu seiner Zeit als legitim und der Übermittlung wert galt.

\subsection{Osmanisches Reich}

Die Bezeichnungen „Türken“ und „Türkei“ verteilen sich über verschiedene Abschnitte der Schulbücher; dabei kann es sich um vereinzelte Erwähnungen oder auch um ausführlichere Darlegungen handeln. Ganze Kapitel oder Unterkapitel, die sich mit dem Osmanischen Reich oder der Türkischen Republik beschäftigen, fehlen dagegen bis auf wenige Ausnahmen. ${ }^{30}$

26 Richert, Hans: Richtlinien für die Lehrpläne der höheren Schulen Preußens. Grundsätzliches und Methodisches. Berlin: Weidmann 1925, S. 64.

27 Richert, Hans: Richtlinien für die Lehrpläne der höheren Schulen Preußens. Lehraufgaben. Berlin: Weidmann 1925, S. 240.

28 Richert: Richtlinien, 1925, S. $241 \mathrm{f}$.

29 Schnabel, Franz: Neueste Zeit. Leipzig: Teubner 1935, Vorwort.

30 Hierbei handelt es sich durchgängig um Darstellungen in Form einer Weltgeschichte, wie beispielsweise Kähler, Friedrich; Ploetz, Karl: Auszug aus der Alten, Mittleren, Neueren und Neuesten Geschichte. Berlin: Ploetz 1928. 


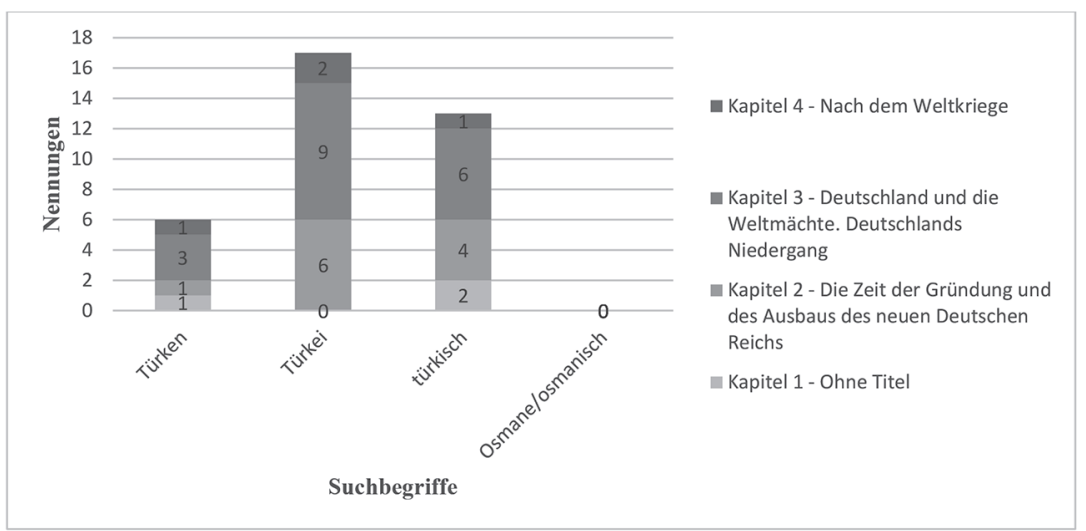

Abb.: Häufigkeitsverteilung bei Neubauer: Vom Wiener Kongress bis zur Gegenwart, 1923.

Das erste Ereignis in den Passagen, in denen von der Türkei oder den Türken die Rede ist, betrifft die Griechische Revolution 1821-1832. ${ }^{31}$ Erzählt wird es wie folgt: Sie brach aus, weil die „Griechen gegen die drückende Herrschaft der Türken“"32 revoltierten. „[M]aßgeblich vorbereitet durch das Unternehmen des Alexander Ypsilanti ${ }^{\text {“33 }}$ war die Erhebung erfolgreich. Mit umfangreicher Unterstützung seitens des ägyptischen „Vizekönigs“ Mehmed Ali gelang es dem Osmanischen Reich zwar, die Rebellion zurückzudrängen. ${ }^{34}$ Aber das Eingreifen englischer, französischer und russischer Truppen ermöglichte es den griechischen Nationalisten, unter der Herrschaft des von den drei Großmächten eingesetzten Königs Otto I. einen Nationalstaat zu gründen. ${ }^{35}$

Wissen wie dieses, also dass 1821 eine griechische Nationalbewegung gegen die osmanische Herrschaft revoltierte und, unterstützt durch die Bewegung des Philhellenismus sowie reguläre russische, englische und französische Armeen, einen

31 Vgl. Zelepos, Ioannis: Griechischer Unabhängigkeitskrieg (1821-1832). 2015, <http:ieg-ego. $\mathrm{eu} / \mathrm{de} / \mathrm{threads} /$ europaeische-medien/europaeische-medienereignisse/ioannis-zelepos-griechischerunabhaengigkeitskrieg-1821-1829> Alle Links in diesem Beitrag wurden zuletzt am 30.03.2020 überprüft. - Zur Darstellung desselben historischen Ereignisses in einem Lehrwerk von 1840 vgl. den Beitrag von Hellmanzik im vorliegenden Band.

32 Neubauer: Vom Wiener Kongreß bis zur Gegenwart für die Mittelstufe, 1923, S. 6.

33 Schnabel: Neueste Zeit, 1924, S. 53.

34 Vgl. Vatikiotis, Panayiotis J.: The history of modern Egypt. From Muhammad Ali to Mubarak. London: Weidenfeld \& Nicolson 1991. Die Bezeichnung „Vizekönig” entstammt zeitgenössischen westlichen Beschreibungen und wird in den Schulbüchern kommentarlos übernommen; vgl. etwa Prokesch-Osten, Anton von: Mehmed-Ali: Vize-König von Aegypten. Aus meinem Tagebuche 1826-1841. Wien: Braumüller 1877.

35 Vgl. Schulz, Oliver: Ein Sieg der zivilisierten Welt? Die Intervention der europäischen Großmächte im griechischen Unabhängigkeitskrieg (1826-1832). Berlin: LIT 2011. 
Nationalstaat gründen konnte, wird als Ereigniswissen gefasst. Es ist als Grundlage einer ereigniszentrierten Darstellung, welcher die Geschichtsschulbücher folgen, über alle untersuchten Werke hinweg konstant. Einbettung, Verknüpfung und Struktur der Erzählung über die Türken unterscheiden sich jedoch und bieten diskursanalytische Ansatzpunkte. So heißt es in Neubauers Version:

„Ein Ereignis, das allenthalben, besonders in Deutschland, mit Begeisterung begrüßt wurde, war die Erhebung der Griechen gegen die drückende Herrschaft der Türken. Man brachte Geld für sie zusammen, Dichter verherrlichten sie, Freiwillige schlossen sich ihren Scharen an [...]. Dennoch hätten die Griechen, seit der Sultan den klugen und mächtigen Vizekönig von Ägypten Mehemed Ali zu Hilfe gerufen und dieser ein Heer und eine Flotte hinübergesandt hatte, ihren Gegnern nicht zu widerstehen vermocht. Aber 1827 mischten sich England, Rußland und Frankreich in den Kampf, und eine vereinigte Flotte dieser Mächte vernichtete die ägyptisch-türkische Flotte in der Bucht von Navarino am Peloponnes. ${ }^{\text {"36 }}$

Schon im ersten Satz wird ein narratives Element angeführt, das der Beachtung bedarf: die „drückende Herrschaft der Türken“. Mit dieser Formulierung soll dem Problem entgegengewirkt werden, dass eine Revolution, die (anderenorts) eine neue Ordnung hervorbringt, womöglich zugleich die Legitimität der eigenen Herrschaft in Frage stellt - ganz im Sinne der aktuellen Transformationsforschung, die für vergleichbare Vorgänge die Delegitimierung der alten Ordnung als durchgängiges Element ermittelt. ${ }^{37}$ Neubauer reproduziert dabei einen Topos, der sich bereits in der Unabhängigkeitserklärung der griechischen Nationalversammlung (1822) und in Schulbüchern jener Epoche findet: ${ }^{38}$ Die osmanischen Herrscher werden als „Räuber" und "grausame Tyrann(en)“ bezeichnet, die „von weit hergekommen“ seien, „um uns gewaltsam zu unterjochen“. Überdies: „Die Verwaltung war schlecht, die Beamten vielfach bestechlich, zu Reformen kam es nicht; einst blühende Kulturgebiete lagen verwahrlost ${ }^{\text {"39 }}$. Aufgegriffen wird damit eine Beschreibung, die in den 1920er Jahren in den vielgelesenen Berichten ehemaliger deutscher Militärs bei der osmanischen Armee verbreitet war. ${ }^{40} \mathrm{Im}$ Mittel-

36 Neubauer: Vom Wiener Kongreß bis zur Gegenwart, 1923, S. 6.

37 Vgl. Lambach, Daniel: Die Empirie der Transformation politischer Ordnung. In: Politische Herrschaft jenseits des Staates: zur Transformation von Legitimität in Geschichte und Gegenwart. Hrsg. von John Emeka Akude. Wiesbaden: VS Verlag für Sozialwissenschaften 2011, S. 259-276, hier 273.

38 Vgl. Hlepas, Nikolaos-Komnenos: Ein romantisches Abenteuer? Nationale Revolution, moderne Staatlichkeit und bayerische Monarchie in Griechenland. In: Ungleichzeitigkeiten der europäischen Romantik. Hrsg. von Alexander von Bormann, Gerhart von Graevenitz. Würzburg: Königshausen \& Neumann 2006, S. 165-204, hier 177, sowie den Beitrag von Hellmanzik im vorliegenden Band.

39 Neubauer: Vom Wiener Kongreß bis zur Gegenwart, 1935, S. 101.

40 Vgl. etwa Schmiterlöw, Bernhard von: Aus dem Leben des Generalfeldmarschalls Freiherr von der Goltz-Pascha: nach Briefen an seinen Freund. Berlin: Koehler 1926; dazu Reichmann, Jan Chri- 
stufenband der Teubner-Reihe finden sich ähnliche Argumentationsmuster; auch hier erhoben die Griechen ,sich gegen die Türken, die sie in harter Unterdrückung gehalten hatten, und behaupteten sich in schweren Kämpfen “41. Implizit wertet somit auch Pinnow die Entwicklung eines griechischen Nationalstaats auf dem Balkan als gerechtfertigte Antwort auf eine ablehnungswürdige Herrschaft des Osmanischen Reichs. Schnabel schließlich ordnet den „griechischen Befreiungskampf“ als ein Ereignis im Rahmen „südeuropäischer Bewegungen“ ein, in welchen nationalliberale Gruppierungen gegen die „reaktionären Regierungen des Kontinentes“ 42 revoltieren. Dabei sei „England dem liberalen Europa von neuem als Vorkämpfer der Freiheit (erschienen)“"43. Über die Ergebnisse des Berliner Kongresses (1878) heißt es, Österreich-Ungarn werde „die Verwaltung von Bosnien und der Herzegowina übertragen, wo die Aufstände zuerst ausgebrochen sind und die türkische Mißwirtschaft in der Tat sehr schlimm gewesen ist " ${ }^{44}$. Auch hier kommt mithin das Narrativ von den schlecht herrschenden Türken zum Einsatz, womit Gegenwehr allemal gerechtfertigt erscheint.

Eingebettet ist die Beschreibung dieser schlechten Herrschaft in die weiter greifende Erzählung vom Niedergang des Osmanischen Reiches mit den Topoi „orientalische Frage“ und „kranker Mann vom Bosporus“. ${ }^{45}$ Gerade das Bild des gebrechlichen alten Mannes, der die Blüte seines Lebens hinter sich hat, hielt sich lange als Stereotyp. Noch in einem deutschen Wörterbuch von 1943 heißt es: „Der kranke Mann (vom Bosporus) ist [ein] geflügeltes Wort für die Türkei“" ${ }^{46}$. Auch in den Geschichtsschulbüchern findet es sich wiederholt. Das Ereignis, welches meist damit verknüpft wird, ist der Krimkrieg $1853-1856 .{ }^{47}$ Schnabel bewertet ihn

stoph: „Tapfere Askers“ und „feige Araber“: der osmanische Verbündete aus der Sicht deutschter Soldaten im Orient 1914-1918. (Diss.) Münster: Westfälische Wilhelms-Universität 2009, S. 52.

41 Pinnow: Von 1648 bis zur Gegenwart, 1923, S. 74.

42 Schnabel: Neueste Zeit, 1924, S. 53.

43 Ebd. S. 53.

44 Schnabel: Neueste Zeit, 1930, S. 78. Zum Berliner Kongress vgl. Yavuz, M. Hakan; Slugett, Peter: War and diplomacy: the Russo-Turkish War of 1877-1878 and the Treaty of Berlin. Salt Lake City: The University of Utah Press 2011.

45 Vgl. zur „orientalischen Frage” grundlegend Schöllgen, Gregor: Imperialismus und Gleichgewicht. Deutschland, England und die orientalische Frage 1871-1914. München: de Gruyter Oldenbourg 2000.

46 Götze, Alfred: I-N. In: Trübners deutsches Wörterbuch. Hrsg. von Walther Mitzka. Berlin: de Gruyter 1943, S. 257. Erzählt wird die Anekdote, wonach Zar Nikolaus I. in einem Gespräch mit Fürst von Metternich das Bild des „kranken Mannes” erstmals zur Beschreibung des Osmanischen Staates nutzte. Zur Wahrnehmung des Osmanischen Reiches in Österreich vgl. Haider-Wilson, Barbara: Tanzimat revisited: Über den Einfluss des Verhältnisses von Orient und Okzident auf die völkerrechtliche Stellung des Osmanischen Reiches im 19. Jahrhundert. In: Orient \& Okzident: Begegnungen und Wahrnehmungen aus fünf Jahrhunderten. Hrsg. von ders., Maximilian Graf. Wien: Neue Welt 2017, S. 405-448.

47 Zum Krimkrieg vgl. Baumgart, Winfried: The Crimean War, 1853-1856. London: Arnold 1999. 
machtpolitisch: „Napoleon III. will, im Sinne der bisherigen französischen Orientpolitik, in der Türkei Einfluß gewinnen und stößt dabei auf Rußland; daneben besteht das alte Ringen zwischen Rußland, das die Türkei zertrümmern will, und England, das sie beschützt: beerben wollen sie beide den ,kranken Mann' „48. Auch Neubauer verweist auf den Gegensatz der außenpolitischen Interessen Russlands und Englands, wenn er schreibt, dass Zar Nikolaus ,auf den Widerstand Englands [stieß], das die Rolle des Beschützers des kranken Mannes am Bosporus spielte“48. In beiden Passagen wird das Narrativ vom Niedergang des Osmanischen Reiches mit den imperialistischen Interessenkonflikten der europäischen Großmächte verknüpft. Schnabel zum Ausgang des Berliner Kongresses: „Und wiederum, wie schon so oft, rettet die Eifersucht der Großmächte den kranken Mann: der Landzuwachs Rußlands und der Balkanstaaten wird durch den Kongreß stark beschnitten “49. Das Niedergangsnarrativ wird an militärischen Niederlagen und territorialen Besitzverlusten festgemacht. Keinerlei Erwähnung finden hingegen die Reformansätze und Entwicklungen des Osmanischen Staates im Rahmen der Tanzimat, jener Neuordnungen, mit denen 1839 begonnen wurde und die mit der Annahme der ersten Verfassung des osmanischen Staates 1876 endeten - und in den deutschen Staaten durchaus auch publizistisch begleitet worden waren. ${ }^{50}$ Dass Vorgänge, die nicht ins Stereotyp passen, gar nicht erst erzählt werden, setzt sich fort. So heißt es bei Neubauer zur innenpolitischen Revolution der Jungtürken: „Da brach (1908) in der Türkei eine Revolution aus, das Werk der jungtürkischen Partei: der Sultan, der bisher mit absoluter Macht regiert und zur Behauptung seiner Herrschaft auch Mord und Gewalttat nicht verschmäht hatte (Hinmordung der Armenier), mußte eine Verfassung geben und bald darauf abdanken; sein Bruder bestieg den Thron, blieb aber ohne jeden Einfluß ${ }^{\text {“51. }}$. Erstmals wird hier osmanische Innenpolitik thematisiert, aber dass bereits 1876 eine Verfassung gegeben worden war, die nun wieder in Kraft gesetzt wurde, wird nicht erwähnt. ${ }^{52}$ Das Geschehen wird allein im Hinblick auf außenpolitische Kräfteverhältnisse gewichtet. Pinnow: „Auf dem Balkan stand Österreich den Ententemächten gegenüber. Als in der Türkei eine Umwälzung stattfand, in deren Verlauf eine Verfassung eingeführt wurde, sprach Österreich die Einverleibung

48 Schnabel: Neueste Zeit, 1924, S. 78.

49 Neubauer, Friedrich: Vom Wiener Kongreß bis zur Gegenwart für die Mittelstufe. Halle an der Saale: Buchhandlung des Waisenhauses 1930, S. 25.

50 Schnabel: Neueste Zeit, 1935, S. 78.

51 Vgl. etwa Petermann, Julius Heinrich; Efendi, Ramis: Beiträge zu einer Geschichte der neuesten Reformen des Osmanischen Reiches. Berlin: Lüderitz 1842; zu den frühen Reformen Scheben, Thomas: Verwaltungsreformen der frühen Tanzimatzeit. Gesetze, Maßnahmen, Auswirkungen. Von der Verkündigung des Ediktes von Gülhane 1839 bis zum Ausbruch des Krimkrieges 1853. Frankfurt am Main: Lang 1991; zur Rezeption Haider-Wilson: Tanzimat revisited, 2017.

52 Neubauer: Vom Wiener Kongreß bis zur Gegenwart, 1923, S. 61. 
der Landschaften Bosnien und Herzegowina aus (1908), die seit 30 Jahren unter österreichischer Verwaltung standen" ${ }^{\text {"53 }}$. Hier wird mit den Ereignissen im Osmanischen Reich eine Entwicklung hin zu einem Verfassungsstaat zwar erwähnt, doch bleibt die Darstellung des Geschehens blass, eine historische Einordnung und Bewertung findet nicht statt. Daneben wird mit dem Begriff „Einverleibung“ die Annexionspolitik des befreundeten Österreichs angesprochen, aber zugleich auch als Nebensächlichkeit behandelt. ${ }^{54}$ Eine vergleichbare Perspektive findet sich in Schnabels Oberstufenbuch. Auch hier wird die jungtürkische Revolution unter dem Gesichtspunkt ihrer begrenzten Nützlichkeit für die außenpolitischen Interessen des Deutschen Kaiserreichs bewertet: „In Ausführung der Politik, die durch die englisch-russische Verständigung eingeleitet wird, zieht sich England von der Türkei langsam zurück. Zunächst erscheint dies als Vorteil Deutschlands, woran auch die jungtürkische Revolution nichts ändert, die den Sturz des deutschfreundlichen Sultans herbeiführt und ein westeuropäisch-konstitutionelles Regi-

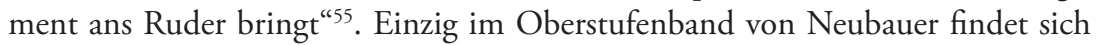
ein Hinweis auf Hintergründe der jungtürkischen Revolution: „1908 brach in der Türkei eine Armeerevolution aus (jungtürkische Partei); Erbitterung über die Ohnmacht nach außen, die Verwahrlosung im Inneren, die Gewaltherrschaft des Sultans waren die Ursachen. Abdul Hamid mußte eine Verfassung verkünden und ein Parlament berufen. " ${ }^{56}$ Der Sturz des Sultans und die Umwälzung des Regierungssystems scheinen nur kurz als möglicher Wendepunkt der Darstellung auf. Im Vordergrund steht das außenpolitisch Wichtige: „Die Türkei fand sich mit der Annexion [von Bosnien und Herzegowina] gegen eine Geldsumme ab. "57

Im Anschluss an die Ereignisse von 1908 widmen sich alle untersuchten Schulbücher der Vorgeschichte des Ersten Weltkriegs einschließlich der internationalen Krisen und Kriege insbesondere auf der Balkanhalbinsel und ihren Auswirkungen

53 Vgl. zum historischen Geschehen Hanioglu, M. Sükrü: The committee of Union and Progress and the 1908 Revolution; Georgeon, François: „L'ivresse de la liberté”: la révolution de 1908 dans l'Empire ottoman. Paris: Peeters 2012; Hanioglu, M. Sükrü: The committee of Union and Progress and the 1908 Revolution. In: „L'ivresse de la liberté": la révolution de 1908 dans l'Empire ottoman. Hrsg. von François Georgeon. Paris: Peeters 2012, S. 15-26.

54 Pinnow: Von 1648 bis zur Gegenwart, 1923, S. 129. Vgl. zur Sicht auf das historische Geschehen in der heutigen Forschung Murakami, Ryo: Die Annexion von Bosnien-Herzegowina und István Burián. In: Prague Papers on the History of International Relations 11.1 (2017), S. 67-89, hier $67 f$.

55 Vgl. Warnke, Ingo: Deutsche Sprache und Kolonialismus. Umriss eines Forschungsfeldes. In: Deutsche Sprache und Kolonialismus. Aspekte der nationalen Kommunikation 1884-1919. Hrsg. von dems. Berlin: de Gruyter 2009, S. 3-64.

56 Schnabel: Neueste Zeit, 1930, S. 110.

57 Neubauer: Vom Wiener Kongreß bis zur Gegenwart für die Oberstufe, 1926, S. 100. 
auf das Osmanische Reich. ${ }^{58}$ Pinnow: „Am schlimmsten erging es den Türken“59. Ähnlich Neubauer:

„Weit verhängnisvoller aber für den Fortbestand des türkischen Reiches wurde es, als (1912) die vier Balkanstaaten Bulgarien, Serbien, Griechenland, Montenegro, durch die russische Diplomatie zu einem Bunde vereinigt, ihre Truppen einrücken ließen: die türkische Armee, schlecht geführt und schlecht verpflegt, wurde allenthalben geschlagen; die tief erschütterte Türkei mußte einen äußerst ungünstigen Frieden abschließen. " ${ }^{\text {"00 }}$

Die Fixierung der Erzählung auf den fortgesetzten Verlust von Territorium und Macht als Niedergang verdrängt jedes andere Narrativ. Dass innenpolitische Veränderungen im Zuge der jungtürkischen Revolution Reformen etwa in Armee und Verwaltung bewirkten, wird nicht zur Sprache gebracht. - Anhand der Darstellung der Balkankriege lassen sich zwei weitere narrative Strukturen herausarbeiten: die Frage der Zugehörigkeit der Türkei zu Europa sowie die Darstellung der sich intensivierenden deutsch-türkischen Beziehungen.

Zunächst zur Europafrage, die bis in die Gegenwart diskutiert wird. ${ }^{61}$ Die in Rede stehenden Schulbuchautoren differenzieren grundsätzlich zwischen europäischen und asiatischen Besitzungen des Osmanischen Reiches, wobei die Meerengen des Bosporus und der Dardanellen als Trennlinien dienen. Schnabel nennt als Ergebnis des ersten Balkankrieges (1912): „Noch einmal rettet dieses Ringen der Mächte wenigstens einen kleinen Rest der europäischen Türkei“"62. Schon als Ergebnis des Russisch-Türkischen Krieges 1877/78 hat er festgehalten, dass „die europäische Türkei fast völlig verschwunden (ist) "63. Auch Neubauer sieht die Türkei zunehmend aus Europa verschwinden: „Da waren es die Ereignisse im Orient,

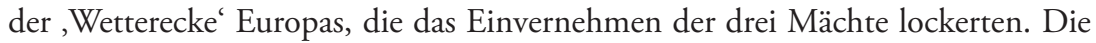
Türkei reichte damals noch bis zur Donau und Save; auch Serbien, Montenegro, Rumänien besaßen noch nicht die volle Unabhängigkeit, sondern leisteten ihr einen Jahrestribut ${ }^{\mathrm{C}} \mathrm{\otimes}$. Die Europafrage wird in den Darstellungen mithin an entsprechende territoriale Besitzungen des Osmanischen Reichs geknüpft. Mit deren Verlusten im späten 19. und frühen 20. Jahrhundert ist von einer Zugehörigkeit der Türkei zu Europa immer seltener die Rede. Der Endpunkt dieser Entwicklung ist die Darstellung der Ergebnisse des Friedensvertrages von Sèvres (1920) nach

58 Vgl. Kießling, Friedrich: Gegen den „großen“ Krieg? Entspannung in den Interantionalen Beziehungen 1911-1914. München: de Gruyter 2002.

59 Pinnow, Hermann: Von 1648 bis zur Gegenwart. Gießen: Roth 1936, S. 126.

60 Neubauer: Vom Wiener Kongreß bis zur Gegenwart, 1923, S. 62.

61 Vgl. König, Helmut; Sicking, Manfred: Statt einer Einleitung: Gehört die Türkei zu Europa? Konturen einer Diskussion. In: Gehört die Türkei zu Europa? Wegweisungen für ein Europa am Scheideweg. Hrsg. von Helmut König. Bielefeld: transcript 2005, S. 9-28.

62 Schnabel: Neueste Zeit, 1930, S. 110.

63 Ebd. S. 77. 
dem Ersten Weltkrieg. Pinnow hierzu: „Die Türkei behielt nur den größten Teil von Kleinasien und Gebiete nördlich des Marmarameeres; Konstantinopel und die Meerengen wurden einer internationalen Kommission unterstellt ${ }^{\text {“64 }}$. In einem größeren zeitlichen Abstand zu den Ereignissen verschwimmen dann die Ergebnisse des Vertrages von Sèvres mit denen von Lausanne (1923): „der Friede von Sèvres [spricht] das Urteil über die Türkei, die auf Konstantinopel und Anatolien beschränkt wird" ${ }^{65}$.

Neben der geographisch-territorialen Verortung des Osmanischen Reiches bestand eine ideengeschichtliche Verwendung des Europabegriffs. Die osmanischen Eroberungen im 15. und 16. Jahrhundert bildeten für die Konstruktion von Europa den notwendigen Kontrast des „Anderen "66 und wurden mit einer intensiven propagandistischen Ausarbeitung der „Türkengefahr ${ }^{\text {“67 }}{ }^{6}$ verknüpft. Entsprechend reichhaltige Nachweise bieten die Schulbücher zu diesem Thema, und zwar sowohl mit Zuordnung des türkischen Reichs zum Konzept „Europa“ als auch mit Abgrenzung davon. Insbesondere in der Darstellung der Griechischen Revolution erhält der semantische Ausschluss des Osmanischen Reiches daraus neue Nahrung: „Die Völker Europas, auch die Deutschen, jubelten ihnen [den Griechen] zu: Dichter verherrlichten ihre Taten, wie z.B. Wilhelm Müller in seinen Liedern der Griechen, Freiwillige und Geldmittel strömten nach Griechenland“68. Andererseits wird das Narrativ vom Niedergang des Osmanischen Reichs zunehmend um die Überlegung ergänzt, ob nicht eine Anlehnung an den Westen, eine Europäisierung, dem Niedergang Einhalt gebieten könnte. In diesem Sinne wird die jungtürkische Regierung als „westeuropäisch-konstitutionelles Regiment“ gewertet. Zwar wird schon als Ergebnis des Krimkriegs herausgestellt, dass „die Unverletzlichkeit des türkischen Reiches gewährleistet ${ }^{\text {"69 }}$ sei und das Osmanische Reich dem „Konzert der europäischen Mächte" ${ }^{\text {“70 }}$ zugehöre, aber im Großen und Ganzen sehen die Schulbücher den Versuch der Anpassung an eine „europäische" kulturelle und politische Ordnung erst in den Abschnitten, in denen das deutsch-türkische Verhältnis erzählt wird.

64 Neubauer: Vom Wiener Kongreß bis zur Gegenwart, 1923, S. 40.

65 Pinnow: Von 1648 bis zur Gegenwart, 1923, S. 146.

66 Schnabel: Neueste Zeit, 1930, S. 134.

67 Schmale, Wolfgang: Geschichte und Zukunft der europäischen Identität. Stuttgart: Kohlhammer 2008, S. $21 \mathrm{f}$.

68 Höfert, Almut: Den Feind beschreiben: „Türkengefahr” und europäisches Wissen über das Osmanische Reich 1450-1600. Frankfurt am Main: Campus 2003. Zur Konstruktion von Europa mittels Beschreibung des „Anderen” Kampmann, Christoph: Einführung. In: Konstruktionen Europas in der Frühen Neuzeit. Hrsg. von Susan Richter, Michael Roth, Sebastian Meurer. Heidelberg: Heidelberg University Publishing 2017, S. 31-39.

69 Pinnow: Von 1648 bis zur Gegenwart, 1936, S. 73.

70 Neubauer: Vom Wiener Kongreß bis zur Gegenwart, 1926, S. 46. 
Diese Erzählung wird speziell mit der Orientpolitik Wilhelms II. verbunden, denn


allem im Militärwesen und in der Ökonomie sei dieses Zusammengehen ausgeprägt gewesen: „Die Türkei ist dem Dreibund [von Deutschland, Österreich-Ungarn und Italien] eng verbunden, weil die österreichischen und türkischen Interessen auf dem Balkan zusammengehen und weil Deutschland in der Türkei starke wirtschaftliche Interessen hat ${ }^{\text {"72 }}$. Als sichtbarstes Zeichen der wirtschaftlichen Anbindung des Osmanischen Reiches an Europa gilt die Bagdadbahn. In den Worten Ernst Jäckhs, eines Protagonisten des deutsch-türkischen Bündnisses, steigert „die Bahn zugleich die Zahl der intelligenten und tüchtigen anatolischen Bauern und deren Steuerkraft und damit die finanzielle und materielle Leistungsfähigkeit des Staates. Und Deutschland schafft sich einen gewinnbringenden Handelsver-


gung an der Bagdadbahn unterbunden hatte, konnte sich zum Beispiel Friedrich Rosen, ehemaliger Außenminister und Vorsitzender der Deutschen Morgenländischen Gesellschaft, eine Wiederaufnahme der deutschen Wirtschaftsunternehmungen durchaus vorstellen. ${ }^{74}$ Ähnliche Argumentationsmuster finden sich in den Schulbüchern für die Zusammenarbeit mit der Türkischen Republik. So fasst Neubauer Mustafa Kemals Wirken 1935 wie folgt zusammen: „Sein Streben ist darauf gerichtet, ein leistungsfähiges Heer zu schaffen, die Verwaltung zu bessern, die Hilfsquellen des Landes zu erschließen, Eisenbahnen zu bauen; dabei sind fremde, auch deutsche Sachkenntnis und Kapital nicht zu entbehren "75.

In ähnlicher Weise erfolgt die Darstellung der militärischen Zusammenarbeit. Mit Blick auf die Niederlage des Osmanischen Reiches im Balkankrieg heißt es: „Ebenso sahen die Türken jetzt ihre einzige Rettung in dem Anschluß an das Deutsche Reich, das ihnen Offiziere schickte, um ihre Armee neu zu gestalten "“76. Der Wert des deutschen Militärs für den osmanischen Staat wird insbesondere für die frühe Phase des Ersten Weltkriegs hervorgehoben: da einerseits den „Russen durch die Sperrung der Dardanellen die Ausfuhr ihres Getreides und die Einfuhr

71 Reinkowski, Maurus: Das Osmanische Reich - ein antikoloniales Imperium? In: Zeithistorische Forschungen/ Studies in Contemporary History 3.1 (2006), S. 34-54, hier 35.

72 Schnabel: Neueste Zeit, 1924, S. 140.

73 Schnabel: Neueste Zeit, 1930, S. 108. Vgl. Fuhrmann, Malte: Deutschlandabenteuer im Orient: Eine Geschichte semi-kolonialer Verstrickungen. In: Türkisch-deutsche Beziehungen. Hrsg. von Claus Schönig. Berlin: Schwarz 2012, S. 10-33.

74 Jäckh, Ernst: Der aufsteigende Halbmond: auf dem Weg zum deutsch-türkischen Bündnis. Stuttgart: Deutsche Verlagsanstalt 1915, S. 55 f.

75 Vgl. Rosen, Friedrich: Deutschlands auswärtige Politik seit dem Vertrage von Versailles. In: Deutschland: Vergangheit und Gegenwart. Bilder zur deutschen Politik und Kulturgeschichte. Hrsg. von Karl Federn, Joachim Kühn. Berlin: Deutscher National-Verlag 1925, S. 57-84, hier $72 \mathrm{f}$.

76 Neubauer: Vom Wiener Kongreß bis zur Gegenwart, 1935, S. 147. 
ihres Kriegsbedarfs unterbunden war" und andererseits die englisch-französischen Truppen „von den Türken, die von dem preußischen General Liman von Sanders befehligt werden, nach langen Kämpfen zur Räumung der Halbinsel (Gallipoli) gezwungen " 77 wurden. Gleichzeitig wird aber auch die Problematik des Verhältnisses thematisiert. So könne nämlich Deutschland seinerseits „nur auf die Hilfe zweier morscher Staaten zählen" "78, Österreich-Ungarns und der Türkei. Und während das deutsche kulturpolitische Engagement im Osmanischen Reich unkritisch gesehen wird, werden damit einhergehende diplomatische und militärische Implikationen durchaus auch skeptisch beurteilt.

Es lässt sich an dieser Stelle festhalten, dass es für die Zuordnung zu Europa geographische Kriterien gab, nach denen die „europäische Türkei“ mit den Balkankriegen nahezu aufhörte zu existieren. Daneben findet sich eine Zuordnung zu Europa anhand zivilisatorischer, wirtschaftlicher und politischer Kriterien. Speziell die Ergebnisse der jungtürkischen Revolution und die deutsche Einflussnahme wurden als Annäherung an Europa gewertet. Die Darstellung dieser beiden Geschehen durchbrach das Narrativ vom Niedergang, welchen die Schulbücher hauptsächlich an territorialen Verlusten und mangelhaften Verwaltungsstrukturen des Osmanischen Reiches festmachten.

\subsection{Türkische Republik}

Im folgenden Abschnitt geht es um die Narrative, mit denen der Türkische Unabhängigkeitskrieg nach dem Ersten Weltkrieg sowie die Türkische Republik bedacht wurden. Der geringe Abstand zwischen Ereignis und Verarbeitungszeitpunkt beeinflusst einerseits die Darstellung in den Schulbüchern, andererseits ist an die Differenz zwischen schnelllebigem massenmedialen Diskurs und der anerkannten Trägheit des Schulbuchwissens zu erinnern. In den Mitteilungen des Bundes der Asienkämpfer wird 1924 - unter dem vielsagenden Titel „Ex oriente lux“ - dem Achtung gezollt, dass es „von den im Weltkriege unterlegenen Mächten allein die Türkei (ist), der gegenüber die Entente-Gewaltigen mit ihrer Methode der Diktate, Reparationen und Sanktionen nicht durchgekommen sind “79. Sie habe „den Gerichtsvollzieher des Ententewillens, Griechenland, aus Kleinasien gewiesen, Frankreich gegen England, England gegen Frankreich ausgespielt und der Welt bewiesen, daß der Kranke Mann am Bosporus, der 1911/12 den Krieg mit Italien, 1912/13 den Krieg mit den Balkanbundesgenossen [...], 1914/18 den Weltkrieg, 1920/22 den Krieg mit dem von England und Frankreich unterstütz-

77 Neubauer: Vom Wiener Kongreß bis zur Gegenwart, 1930, S. 73.

78 Schnabel: Neueste Zeit, 1924, S. 155.

79 Neubauer: Vom Wiener Kongreß bis zur Gegenwart, 1926, S. 102. 
ten Griechenland durchgemacht hatte, durch die Stahlkuren, die ihm seine westmächtlichen Ärzte verordneten, anscheinend gesund geworden ist. ${ }^{\text {"80 }}$

Begründet wird dieser Erfolg damit, dass "die Türkei wieder einen Führer" ${ }^{\text {"81 }}$ besessen habe, und die Hoffnung geäußert, dass sich eine ähnliche Wandlung auch in Deutschland einstellen möge.

In den Erstausgaben der hier untersuchten Schulbuchreihen endet die Darstellung mit dem Vertrag von Sèvres. Erst 1926, nach der Einführung der neuen Lehrpläne in Preußen (1925), werden die Nachkriegsereignisse in den europäischen Staaten skizziert. Zu diesen wird die Türkische Republik nicht mehr gezählt, zumal der Türkische Unabhängigkeitskrieg bewirkte, dass „Griechenland, dessen Truppen noch im Frühjahr 1922 tief in Kleinasien standen, durch den siegreichen türkischen Ansturm nicht nur dieses Land ganz eingebüßt, sondern auch Thrazien verloren (hat) ${ }^{\text {“82 }}$. Diese erneute geographische Zuordnung zu Asien findet sich auch bei Schnabel, der den Türkischen Unabhängigkeitskrieg - bemerkenswerterweise im Kapitel „Das Erwachen der kolonialen Völker" - in einem Abschnitt „Die mohammedanische Welt" behandelt. ${ }^{83}$ Gleichzeitig deutet sich das Ende des Narrativs vom Niedergang an, den der „siegreiche türkische Ansturm“ bewirken könnte: „Die Türkei hat, unter der Führung von Mustafa Kemal Pascha, eine außerordentliche Wandlung durchgemacht. [...] Während sie früher große Mengen arabischer, griechischer, slawischer Untertanen hatte, ist sie jetzt ein Nationalstaat geworden" ${ }^{84}$. Das Aufgreifen der nationalpolitisch motivierten Umsiedelung ganzer Ethnien nach dem Ersten Weltkrieg und vor allem der Vertrag von Lausanne schlossen an einen Diskurs an, den speziell völkisch-nationale Beobachter der Entwicklungen in der Türkei führten. Hier werden die Zwangsumsiedlungen lobend erwähnt, ${ }^{85}$ und es wird festgehalten, dass die Türkei, um ein Nationalstaat werden zu können, die anderen Bevölkerungsgruppen aus diesem Staat habe ausschließen müssen. ${ }^{86}$ Diese Argumentation wird nach 1933 auch zur Erklärung des Niedergangs des Osmanischen Reiches mitherangezogen: „Die Türkei war, so wenig wie Österreich-Ungarn, ein Nationalstaat; von den kaum 20 Millionen Einwohnern [...] war kaum die Hälfte Osmanen [...]. Der Kern der Bevölkerung, zugleich treffliche Soldaten, waren die anatolischen Bauern" ${ }^{\text {"87. }}$

80 Pritze, Johannes: Ex oriente lux. Mitteilungen des Bundes der Asienkämpfer 6.9 (1924), S. 3.

81 Ebd.

82 Pritze, Johannes: Der neue Geist. Mitteilungen des Bundes der Asienkämpfer 5.4 (1923), S. 1.

83 Neubauer: Vom Wiener Kongreß bis zur Gegenwart, 1926, S. 126.

84 Vgl. Schnabel, Neueste Zeit, 1930, S. 154.

85 Neubauer: Vom Wiener Kongreß bis zur Gegenwart für die Oberstufe, 1929, S. 147.

86 Vgl. Tröbst, Hans: Mustafa Kemal Pascha und sein Werk (VI). Heimatland (1923), 15. Oktober; Ihrig: Atatürk in the Nazi Imagination, 2014, S. 68ff.

87 Vgl. Die Stellung der neuen Türkei. Hamburger Tageblatt (1935), 5. Mai. 
Als große Errungenschaft wird daneben die Trennung von staatlichen und religiösen Institutionen im Zuge der säkularisierungspolitischen Maßnahmen der Türkischen Republik bewertet: „die Türkei ist ein rein weltlicher Staat “88. Die Säkularisierung wird durchweg als Merkmal einer positiven Entwicklung bewertet, ${ }^{89}$ insbesondere das Verbot von Fez und Schleier wird als Zeichen einer Europäisierung des asiatischen Staates gedeutet. ${ }^{90} 1935$ wird lobend hinzugefügt, dass nun auch Frauen das Wahlrecht besäßen. ${ }^{91}$ Deutlicher denn je wird unterstrichen, dass die territorialen Entwicklungen eine Einordnung der Türkei als asiatischen Staat notwendig machten, dass gleichzeitig aber die Reformen eine Zuordnung zu Europa wieder möglich erscheinen ließen. In der Tagespresse werden neben der Beseitigung religiöser Bekleidungsvorschriften unter der Überschrift „Die Europäisierung der Türkei“ auch die Implementierung des schweizerischen bürgerlichen Rechts ${ }^{92}$ sowie des italienischen Strafrechts und die Einführung der lateinischen Schriftzeichen hervorgehoben. ${ }^{93}$ Die Frage der Integration der Türkei als Peripherie eines als europäisch verstandenen Raums setzt sich bis in die Gegenwart fort. ${ }^{94}$ Es bleibt festzuhalten, dass die Türkische Republik, ihre Entstehung und Entwicklung in den untersuchten Geschichtsschulbüchern eigens behandelt wurden, entweder wie bei Neubauer in eigenen kurzen Abschnitten oder wie bei Schnabel im Rahmen eines größeren historisch-politischen Zusammenhangs. Auffällig ist, dass die prägenden Narrative der Erstausgaben in den späteren Auflagen präsent blieben - einschließlich der Erzählung der „zwei Türkei“, also der Unterscheidung einer europäischen von einer asiatischen, wobei die Türkische Republik letzterer zugerechnet wird. Weitergetragen wird auch das Narrativ von der Europäisierung, das erst die jungtürkische Revolution und die deutsche Kulturmission mit den Reformen in der Türkei in Verbindung bringt.

\section{Schluss}

Im Sinne der eingangs gestellten Frage lassen sich vier narrative Strukturen als zentral nachweisen. Grundlegend für die Darstellung der Geschichte des Osmanischen Reiches ist hauptsächlich die Narration seines Niedergangs. Dieser manifestiert sich für die Autoren anhand des schwindenden territorialen Besitzes als Machtfaktor. Reformen, die während der Tanzimat-Ära bis zu einer ersten

88 Neubauer: Vom Wiener Kongreß bis zur Gegenwart für die Mittelstufe, 1935, S. 101.

89 Neubauer: Vom Wiener Kongreß bis zur Gegenwart, 1929, S. 147.

90 Vgl. Ihrig: Atatürk in the Nazi Imagination, 2014.

91 Vgl. Neubauer: Vom Wiener Kongreß bis zur Gegenwart, 1929, S. 147.

92 Vgl. Neubauer: Vom Wiener Kongreß bis zur Gegenwart, 1935, S. 147.

93 Vgl. den Beitrag von Hoffmann-Ocon und Grube im vorliegenden Band.

94 Vgl. etwa Die Europäisierung der Türkei. Deutsche Allgemeine Zeitung (1926), 3. März. 
Verfassung führten, fehlen in der Darstellung - ein Beispiel für diskursives Nichtgesagtes - und das Osmanische Reich wird teleologisch hin bis zum auf Zentralanatolien beschränkten Staat nach dem Ersten Weltkrieg entwickelt. Ansätze eines Abweichens von diesem beherrschenden Narrativ lassen sich hinsichtlich der jungtürkischen Revolution erkennen. Aber erst die Erfolge der Türkischen Republik bei der Revision des Vertrages von Sèvres führen zur Durchbrechung des Niedergangstopos. Eng hiermit verbunden sind die weiteren Narrative. So findet sich in allen drei Reihen eine negative Beurteilung der Herrschaftspraxis des Osmanischen Reiches, insbesondere auf der Balkanhalbinsel; Neubauer beispielsweise spricht quer durch alle Auflagen von „drückender Herrschaft“ und „Türkenjoch“. ${ }^{55}$ Diese Bewertung steht in engem Zusammenhang damit, ob das Osmanische Reich beziehungsweise die Türkei als zugehörig zu Europa angesehen wird oder nicht. Alle Autoren unterscheiden anhand geographischer Merkmale einhellig einen europäischen von einem asiatischen Teil - mit der Folge, dass die Türkische Republik als asiatischer Staat eingeordnet wird. Gleichzeitig ist das Narrativ „Europa“ auch das Zivilisationskriterium. Die Reformen von 1908 und im noch stärkeren Maße die Säkularisierung, die Konstruktion eines ethnisch homogenen Nationalstaats und die Bildungsreformen der Türkischen Republik werden als Fortschreiten eines Zivilisierungsprozesses, als „Europäisierung“ bezeichnet, an dessen Ende eine Zuordnung der Türkei zu Europa, dem Zentrum der Zivilisation, stehen könnte. Ein erster Schritt in diese Richtung sehen die Schulbücher nicht in den Tanzimat und der osmanischen Verfassung von 1876, sondern im deutsch-türkischen Bündnis vor und während des Ersten Weltkriegs. Diese Erzählung wird grundsätzlich von einer positiven Einschätzung der „Kulturmission "966 beherrscht.

Selbstverständlich ergeben die untersuchten Schulbuchreihen kein vollständiges Bild dessen, was zwischen 1919 und 1936 über „die Türken“ und „die Türkei“ aus pädagogischer Sicht als übermittlungswürdiges und aus staatlicher Sicht als legitimes Wissen galt. Immerhin jedoch sind alle drei Reihen aufgrund ihrer hohen Auflagenstärke als viel genutzte und weit verbreitete Schulbücher zu identifizieren. Da für die vorliegende Untersuchung nur solche Geschichtsschulbücher herangezogen wurden, die den Zeitraum ab 1815 darstellen, erscheint die „Türkei“ erstmals während der Griechischen Revolution 1821-1832. Der nächste Auftritt erfolgt erst mit dem Krimkrieg von 1853 bis 1856, also rund 30 Jahre später. Neben der Griechischen Revolution und dem Krimkrieg verdichtet sich das Ereigniswissen mit dem verlustreichen Russisch-Türkischen Krieg von 1877/78 bis

95 Vgl. Gehring, Axel: Vom Mythos des starken Staates und der europäischen Integration der Türkei. Über eine Ökonomie an der Peripherie des euro-atlantischen Raumes. Wiesbaden: VS Verlag für Sozialwissenschaften 2019.

96 Vgl. Koller, Christian: Fremdherrschaft. Ein politischer Kampfbegriff im Zeitalter des Nationalismus. Frankfurt am Main: Campus 2005, S. 339ff. 
hin zum Ausbruch des Ersten Weltkriegs. Hier wird mit der jungtürkischen Revolution - erstmals - auch ein innenpolitisches Thema behandelt, wenn auch in erster Linie im Hinblick auf die außenpolitischen Konsequenzen. Außerdem findet sich in allen drei Reihen eine Darstellung der Annäherung zwischen Deutschem Reich und Osmanischem Reich im Vorfeld des Weltkriegs. Schließlich enden die Erstausgaben der Schulbücher mit den Pariser Vorortverträgen (1919/20), während in den späteren Auflagen eine, wenn auch meist sehr kurze, Beschreibung der Türkischen Republik das letzte Ereigniswissen liefert.

Hinsichtlich dieser inhaltlichen Begrenzung bleibt noch zu beantworten, ob sich die Narrative im Untersuchungszeitraum wandeln. Für die weiter in der Vergangenheit liegenden Ereignisse ist dies zu verneinen, da sowohl das Ereigniswissen als auch die verbindenden Narrative konstant bleiben. Ein Wandel der Zuschreibungen ist erst in der Darstellung der Türkischen Republik zu verzeichnen. Erst hier erscheint „die Türkei“ - zumindest bis Mitte der 1930er Jahre tatsächlich in einem neuen Licht, das neue Wege der Wahrnehmung, etwa der Europäisierung, möglich scheinen lässt.

\section{Quellen und Literatur}

Baumgart, Winfried: The Crimean War, 1853-1856. London: Arnold 1999.

Blänsdorf, Agnes: Lehrwerke für Geschichtsunterricht an Höheren Schulen 1933-1945. Autoren und Verlage unter den Bedingungen des Nationalsozialismus. In: Nationalsozialismus in den Kulturwissenschaften 1: Fächer Milieus Karrieren. Hrsg. von Hartmut Lehmann, Otto Gerhard Oexle. Göttingen: Vandenhoeck \& Ruprecht 2004, S. 273-370.

Bonwetsch, Gerhard: Mittelalter. Leipzig: Teubner 1924.

Bonwetsch, Gerhard; Kania, Hans: Neuzeit. Leipzig: Teubner 1924.

Böttcher, Julika; Kesper-Biermann, Sylvia; Lohmann, Ingrid; Mayer, Christine: Deutsch- türkischer Bildungsraum um 1918 - Akteure, Visionen und Transformationen. In: Jahrbuch für Historische Bildungsforschung 25 (2019), S. 114-159.

Die Europäisierung der Türkei. Deutsche Allgemeine Zeitung (1926), 3. März.

Die Stellung der neuen Türkei. Hamburger Tageblatt (1935), 5. Mai.

Fuhrmann, Malte: Deutschlandabenteuer im Orient: Eine Geschichte semi-kolonialer Verstrickungen. In: Türkisch-deutsche Beziehungen. Hrsg. von Claus Schönig. Berlin: Schwarz 2012, S. 10-33.

Gehring, Axel: Vom Mythos des starken Staates und der europäischen Integration der Türkei. Über eine Ökonomie an der Peripherie des euro-atlantischen Raumes. Wiesbaden: Springer VS 2019.

Götze, Alfred: I-N. In: Trübners deutsches Wörterbuch. Hrsg. von Walther Mitzka. Berlin: de Gruyter 1943.

Guillemarre-Acet, Dorothée: Impérialisme et nationalisme. L'Allemagne, l'Empire ottoman et la Turquie (1908-1933). Würzburg: Ergon 2009.

Haider-Wilson, Barbara: Tanzimat revisited: Über den Einfluss des Verhältnisses von Orient und Okzident auf die völkerrechtliche Stellung des Osmanischen Reiches im 19. Jahrhundert. In: Orient \& Okzident: Begegnungen und Wahrnehmungen aus fünf Jahrhunderten. Hrsg. von ders. und Maximilian Graf. Wien: Neue Welt 2017, S. 405-448.

Hanioglu, M. Sükrü: The committee of Union and Progress and the 1908 Revolution. In: „L'ivresse de la liberté“. La révolution de 1908 dans l'Empire ottoman. Hrsg. von François Georgeon. Paris: Peeters 2012, S. 15-26. 


\section{Dennis Mathie}

Hellmanzik, Timm G.: „Als Brüder sind die Türken niemals betrachtet, auch der europäischen Geistesbildung immer fremd geblieben“ - Das Türkenbild in Joseph Annegarns Weltgeschichte für die katholische Jugend (1840). (Im vorliegenden Band).

Hertfelder, Thomas: Franz Schnabel und die deutsche Geschichtswissenschaft. Geschichtsschreibung zwischen Historismus und Kulturkritik (1910-1945). Göttingen: Vandenhoeck \& Ruprecht 1998.

Hlepas, Nikolaos-Komnenos: Ein romantisches Abenteuer? Nationale Revolution, moderne Staatlichkeit und bayerische Monarchie in Griechenland. In: Ungleichzeitigkeiten der europäischen Romantik. Hrsg. von Alexander von Bormann und Gerhart von Graevenitz. Würzburg: Königshausen \& Neumann 2006, S. 165-204.

Höfert, Almut: Den Feind beschreiben: „Türkengefahr“ und europäisches Wissen über das Osmanische Reich 1450-1600. Frankfurt am Main: Campus 2003.

Hoffmann-Ocon, Andreas; Grube, Norbert: „Wo die Parteien sich mit Staatsstreichen, Militärrevolten, Hinrichtungen und Morden bekämpfen" - Türkeibilder in schweizerischen Geschichtslehrmitteln in der ersten Hälfte des 20. Jahrhunderts. (Im vorliegenden Band).

Höhne, Thomas: Schulbuchwissen. Frankfurt am Main: Johann Wolfgang Goethe-Universität 2003.

Ihrig, Stefan: Atatürk in the Nazi imagination. Cambridge: The Belknap Press of Harvard University Press 2014.

Jäckh, Ernst: Der aufsteigende Halbmond: auf dem Weg zum deutsch-türkischen Bündnis. Stuttgart: Deutsche Verlagsanstalt 1915.

Jacobmeyer, Wolfgang: Das deutsche Schulgeschichtsbuch 1700-1945. Die erste Epoche seiner Gattungsgeschichte im Spiegel der Vorworte. Berlin: LIT 2011.

Kähler, Friedrich; Ploetz, Karl: Auszug aus der Alten, Mittleren, Neueren und Neuesten Geschichte. Berlin: A. G. Ploetz 1928.

Kampmann, Christoph: Einführung. In: Konstruktionen Europas in der Frühen Neuzeit. Hrsg. von Susan Richter, Michael Roth, Sebastian Meurer. Heidelberg: Heidelberg University Publishing 2017, S. 31-39.

Keller, Reiner: Diskursforschung. Eine Einführung für SozialwissenschaftlerInnen. Wiesbaden: VS Verlag für Sozialwissenschaften 2011.

Keller, Reiner: Wissenssoziologische Diskursanalyse. In: Handbuch sozialwissenschaftliche Diskursanalyse. Hrsg. von Reiner Keller. Wiesbaden: VS Verlag für Sozialwissenschaften 2011, S. 125-158.

Kießling, Friedrich: Gegen den "großen“ Krieg? Entspannung in den internationalen Beziehungen 1911-1914. München: de Gruyter 2002.

Koller, Christian: Fremdherrschaft. Ein politischer Kampfbegriff im Zeitalter des Nationalismus. Frankfurt am Main: Campus 2005.

König, Helmut und Sicking, Manfred: Statt einer Einleitung: Gehört die Türkei zu Europa? Konturen einer Diskussion. In: Gehört die Türkei zu Europa? Wegweisungen für ein Europa am Scheideweg. Hrsg. von Helmut König. Bielefeld: transcript 2005, S. 9-28.

Kreiser, Klaus: Der osmanische Staat 1300-1922. München: Oldenbourg 2008.

Kreusch, Julia: Der Schulbuchverlag. In: Geschichte des deutschen Buchhandels im 19. und 20. Jahrhundert. Die Weimarer Republik 1918-1933. Hrsg. von der Historischen Kommission des Börsenvereins des Deutschen Buchhandels. Berlin, Boston: de Gruyter 2012, S. 219-240.

Kreusch, Julia: Der Verlag der Buchhandlung des Waisenhauses als Schulbuchverlag zwischen 1830 und 1918. Die erfolgreichen Geografie- und Geschichtslehrbücher und ihre Autoren. Halle: Verlag der Franckeschen Stiftungen im Max Niemeyer Verlag 2008.

Lambach, Daniel: Die Empirie der Transformation politischer Ordnung. In: Politische Herrschaft jenseits des Staates: zur Transformation von Legitimität in Geschichte und Gegenwart. Hrsg. von John Emeka Akude. Wiesbaden: VS Verlag für Sozialwissenschaften 2011, S. 259-276.

Landwehr, Achim: Historische Diskursanalyse. Frankfurt am Main: Campus 2009. 
Leonhard, Jörn: Der überforderte Frieden: Versailles und die Welt 1918-1923. München: C.H. Beck 2018.

Link, Jürgen: Diskurs, Interdiskurs, Kollektivsymbolik. Am Beispiel der aktuellen Krise der Normalität. Zeitschrift für Diskursforschung 1.1 (2013), S. 7-23.

Mangold-Will, Sabine: Begrenzte Freundschaft: Deutschland und die Türkei 1918-1933. Göttingen: Wallstein 2013.

Ministerium der geistlichen Unterrichts- und Medizinalangelegenheiten: Ordnung für die Einführung von Lehrbüchern an den höheren Lehranstalten für die männliche und für die weibliche Jugend. Berlin: Zentralblatt für die gesamte Unterrichtsverwaltung 1913.

Murakami, Ryo: Die Annexion von Bosnien-Herzegowina und István Burián. Prague Papers on the History of International Relations 11.1 (2017), S. 67-89.

Neubauer, Friedrich: Deutsche Geschichte von der Gründung des nationalen Staats bis zum Westfälischen Frieden. Halle an der Saale: Buchhandlung des Waisenhauses 1923.

Neubauer, Friedrich: Deutsche Geschichte. Halle an der Saale: Buchhandlung des Waisenhauses 1924.

Neubauer, Friedrich: Geschichte des Altertums und des frühen Mittelalters für die Mittelstufe. Halle an der Saale: Buchhandlung des Waisenhauses 1923.

Neubauer, Friedrich: Geschichte des Altertums und des frühen Mittelalters für die Oberstufe. Halle an der Saale: Buchhandlung des Waisenhauses 1923.

Neubauer, Friedrich: Vom Beginn des 19. Jahrhunderts bis zur Gegenwart für die Oberstufe. Halle an der Saale: Buchhandlung des Waisenhauses 1924.

Neubauer, Friedrich: Vom Westfälischen Frieden bis zum Wiener Kongreß. Halle an der Saale: Buchhandlung des Waisenhauses 1923.

Neubauer, Friedrich: Vom Wiener Kongreß bis zur Gegenwart für die Mittelstufe. Halle an der Saale: Buchhandlung des Waisenhauses 1923, 1930 und 1935.

Neubauer, Friedrich: Vom Wiener Kongreß bis zur Gegenwart für die Oberstufe. Halle: Buchhandlung des Waisenhauses 1926 und 1929.

Neubauer, Friedrich: Vom Wiener Kongreß bis zur Gegenwart. Halle (Saale): Buchhandlung des Waisenhauses 1935.

Neubauer, Friedrich: Von der Gründung des deutschen nationalen Staats bis zum Ende des 18. Jahrhunderts. Halle an der Saale: Buchhandlung des Waisenhauses 1924.

Neustadt, Ernst; Röhm, Georg: Altertum. Leipzig: Teubner 1924.

Petermann, Julius Heinrich; Ramis Efendi: Beiträge zu einer Geschichte der neuesten Reformen des Osmanischen Reiches. Berlin: Lüderitz 1842.

Pinnow, Hermann: Geschichte des deutschen Volkes von 1648 bis zur Gegenwart. Leipzig: Teubner 1923.

Pinnow, Hermann: Geschichte des deutschen Volkes von den Anfängen bis 1648. Leipzig: Teubner 1923.

Pinnow, Hermann: Von 1648 bis zur Gegenwart. Gießen: Roth 1936.

Pinnow, Hermann; Bux, Ernst: Geschichte des Altertums. Leipzig: Teubner 1923.

Pritze, Johannes: Der neue Geist. Mitteilungen des Bundes der Asienkämpfer 5.4 (1923), S. 1.

Pritze, Johannes: Ex oriente lux. Mitteilungen des Bundes der Asienkämpfer 6.9 (1924), S. 3.

Prokesch-Osten, Anton von: Mehmed-Ali: Vize-König von Aegypten. Aus meinem Tagebuche 18261841. Wien: Braumüller 1877.

Reichmann, Jan Christoph: „Tapfere Askers“ und „feige Araber“: der osmanische Verbündete aus der Sicht deutschter Soldaten im Orient 1914-1918. (Diss.) Münster: Westfälische Wilhelms-Universität 2009.

Reinkowski, Maurus: Das Osmanische Reich ein antikoloniales Imperium? Zeithistorische Forschungen/ Studies in Contemporary History 3.1 (2006), S. 34-54. 


\section{Dennis Mathie}

Richert, Hans: Richtlinien für die Lehrpläne der höheren Schulen Preußens. Grundsätzliches und Methodisches. Berlin: Weidmann 1925.

Richert, Hans: Richtlinien für die Lehrpläne der höheren Schulen Preußens. Lehraufgaben. Berlin: Weidmann 1925.

Riedler, Florian: Nationalismus und internationale Sensibilität. Transnationale Akteure und die deutsch-türkischen Beziehungen in der Zwischenkriegszeit. In: Aufbruch ins postkoloniale Zeitalter. Hrsg. von Sönke Kunkel, Christoph Meyer. Frankfurt am Main: Campus 2012, S. 251-274.

Rosen, Friedrich: Deutschlands auswärtige Politik seit dem Vertrage von Versailles. In: Deutschland: Vergangenheit und Gegenwart. Bilder zur deutschen Politik und Kulturgeschichte. Hrsg. von Karl Federn, Joachim Kühn. Berlin: Deutscher National-Verlag 1925, S. 57-84.

Scheben, Thomas: Verwaltungsreformen der frühen Tanzimatzeit. Gesetze, Maßnahmen, Auswirkungen. Von der Verkündigung des Ediktes von Gülhane 1839 bis zum Ausbruch des Krimkrieges 1853. Frankfurt am Main: Peter Lang 1991.

Schellberg, Wilhelm; Hylla, Erich: Die Bestimmungen über Einführung von Lehrbüchern. Berlin: Weidmannsche Buchhandlung 1926.

Schiefer, Wilhelm; Hohmann, Walter; Maier, Albert; Schirmeyer, Ludwig: Deutsche Geschichte vom Spätmittelalter (1250) bis zum Wiener Kongreß (1815). Frankfurt am Main: Diesterweg 1935.

Schmale, Wolfgang: Geschichte und Zukunft der europäischen Identität. Stuttgart: Kohlhammer 2008.

Schmiterlöw, Bernhard von: Aus dem Leben des Generalfeldmarschalls Freiherr von der Goltz-Pascha: nach Briefen an seinen Freund. Berlin: Koehler 1926.

Schnabel, Franz: Neueste Zeit. Leipzig: Teubner 1924, 1930, 1935.

Schöllgen, Gregor: Imperialismus und Gleichgewicht. Deutschland, England und die orientalische Frage 1871-1914. München: de Gruyter Oldenbourg 2000.

Schulz, Oliver: Ein Sieg der zivilisierten Welt? Die Intervention der europäischen Großmächte im griechischen Unabhängigkeitskrieg (1826-1832). Berlin: LIT 2011.

Tröbst, Hans: Mustafa Kemal Pascha und sein Werk (VI). Heimatland (1923), 15. Oktober.

Ullrich, Sebastian: Mehr Schall als Rauch. Der Streit um den Namen der ersten deutschen Demokratie 1918-1949. In: Die „Krise“ der Weimarer Republik. Zur Kritik eines Deutungsmusters. Hrsg. von Moritz Föllmer. Frankfurt am Main: Campus 2005, S. 187-208.

Vatikiotis, Panayiotis J.: The history of modern Egypt. From Muhammad Ali to Mubarak. London: Weidenfeld \& Nicolson 1991.

Viehöver, Willy: Diskurse als Narrationen. In: Handbuch sozialwissenschaftliche Diskursanalyse 1: Theorien und Methoden. Hrsg. von Reiner Keller. Wiesbaden: VS Verlag für Sozialwissenschaften 2006, S. 179-208.

Vom Bruch, Rüdiger: Weltpolitik als Kulturmission. Auswärtige Kulturpolitik und Bildungsbürgertum in Deutschland am Vorabend des Ersten Weltkrieges. Paderborn: Schöningh 1982.

Warnke, Ingo: Deutsche Sprache und Kolonialismus. Umriss eines Forschungsfeldes. In: Deutsche Sprache und Kolonialismus. Aspekte der nationalen Kommunikation 1884-1919. Hrsg. von dems. Berlin: de Gruyter 2009, S. 3-64.

White, Hayden: The Value of Narrativity in the Representation of Reality. Critical Inquiry 7.1 (1980), S. 5-27.

Yavuz, M. Hakan; Slugett, Peter: War and diplomacy: the Russo-Turkish War of 1877-1878 and the Treaty of Berlin. Salt Lake City: The University of Utah Press 2011.

Zelepos, Ioannis: Griechischer Unabhängigkeitskrieg (1821-1832). Mainz: Leibniz-Institut für Europäische Geschichte 2015. 